\title{
最小邻域均值投影函数及其在眼睛定位中的应用
}

\author{
郑 颖，汪增福
}

(中国科学技术大学 自动化系,安徽 合肥 230027)

\section{Minimal Neighborhood Mean Projection Function and Its Application to Eye Location}

\author{
ZHENG Ying, WANG Zeng-Fu ${ }^{+}$ \\ (Department of Automation, University of Science and Technology of China, Hefei 230027, China) \\ + Corresponding author: E-mail: zfwang@ustc.edu.cn
}

Zheng Y, Wang ZF. Minimal neighborhood mean projection function and its application to eye location. Journal of Software, 2008,19(9):2322-2328. http://www.jos.org.cn/1000-9825/19/2322.htm

\begin{abstract}
A projection function called minimal neighborhood mean projection function (MNMPF) is proposed. The projection function calculates and stores the minimal neighborhood mean of each pixel on each projection line, so that it is able to trace the low grayscale features in image. Compared with traditional projection functions, i.e. integral projection function (IPF) and variance projection function (VPF), MNMPF is insensitive to sheet noise, due to the local selectivity of its minimum operation. During the computation of MNMPF, the image locations of minima are recorded at the same time. This makes MNMPF a 2D operator. All these properties of MNMPF are very suitable for eye location. It can bring precise and robust response to eyes, especially pupils. Experiments on CAS-PEAL and BioID databases show its excellent correct rate and precision over traditional projection functions.
\end{abstract}

Key words: projection function; eye location; eigeneyes; CAS-PEAL; BioID

摘 要: 提出一种投影函数: 最小邻域均值投影函数. 该函数通过计算每条投影线上各像素点邻域均值的最小值 来跟踪图像中的低灰度特征.与传统的积分投影函数和方差投影函数相比, 它以求最小值的局部选择性代替传统投 影函数的全局累加性,因此具有对片状喿声不敏感的特点,此外,在计算过程中, 它还能记录最小值点的二维位置信 息,是一个二维的搜索算子.最小邻域均值投影函数的这些特点使其非常适合于眼睛定位.它对眼睛,特别是瞳孔,总 能够产生精确、鲁棒的响应.通过在 CAS-PEAL 数据库和 BioID 数据库上的实验表明,其定位正确率与精确度均高 于传统的投影函数.

关键词: 投影函数;眼睛定位;特征眼;CAS-PEAL;BioID

中图法分类号: TP391 文献标识码: A

投影是一种简单、有效的数字图像处理方法.投影将一幅二维图像沿垂直于某一投影轴的方向(也称为投 影线),使用所定义的投影函数,映射成该投影轴上的一个一维序列,从而达到特征选择及降维的目的.选取不同

* Supported by the Opening Found of National Laboratory of Pattern Recognition of Institute of Automation, the Chinese Academy of Sciences (中国科学院自动化研究所模式识别国家重点实验室开放基金); the Graduate Innovation Fund of USTC under Grant No.KD2006042 (中国科学技术大学研究生创新基金)

Received 2006-10-10; Accepted 2007-03-26 
的投影轴进行投影,可以分析图像在不同方向上的分布特征.典型的投影轴的选取可以是图像坐标系的横轴与 纵轴,此时,相应的投影序列分别被称为垂直投影和水平投影. 而通过选取不同的投影函数,可以刻画图像在投 影线上的不同性质.常用的投影函数有积分投影函数和方差投影函数.

投影在人脸识别领域有着广泛的应用,是重要的人脸图像预处理和特征提取手段.早期的应用主要是利用 积分投影函数定位人脸和分割面部特征 ${ }^{[1,2]}$.Feng 和 Yuen ${ }^{[3]}$ 提出方差投影函数的概念并将其应用于眼睛定位. Kumar 等人 ${ }^{[4]}$ 则是利用积分和方差投影函数,通过对眼睛的不同响应来定位眼睛窗口.耿新等人 ${ }^{[5]}$ 结合这两种 投影函数各自的特点,进一步提出了混合投影函数,据此了解图像灰度均值和方差的综合变化情况.

眼睛是人脸上的显著特征. 眼睛的精确定位有利于人脸配准和特征提取,对提高人脸识别正确率有着重要

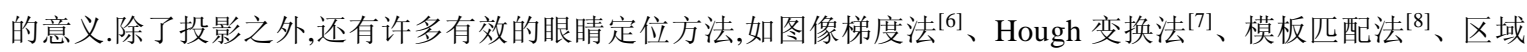
增长法 ${ }^{[9]}$ 等.而若干种方法的结合往往能给出令人满意的定位效果. $\mathrm{Xu}$ 和 $\mathrm{Shi}^{[10]}$ 利用积分投影给出眼睛位置的 初始估计,再用加权 Hough 变换进行细定位. $\mathrm{Du}^{[11]}$ 则是采用谷检测算法和人脸对称性获得初始估计,然后,采用 缺失估计 (lacunarity estimation)进行篮选.小波作为一种图像特征提取方法也被用于眼睛定位.Huang 和 Wechsler ${ }^{[12]}$ 利用最优小波包提取眼睛特征,并利用径向基函数分类眼睛与非眼睛区域.Feris ${ }^{[13]}$ 等人采用两层 Gabor 小波神经网络来完成人脸粗定位和眼睛精确定位的工作.Gabor 小波还被用来处理眼睛定位中光照变化 的问题 ${ }^{[14]}$.还有一些学者将眼睛定位看作是一个典型的模式分类问题,即区分眼睛和非眼睛区域.一些模式分类 的方法,如 Boosting,SVM 等,也被用于眼睛定位 ${ }^{[15-18]}$.关于此类方法的介绍和对比,参见文献[19].

本文提出一种新的投影函数:最小邻域均值投影函数,并将其用于眼睛定位. 本文第 1 节给出已有的投影函 数的定义. 第 2 节在分析这些传统投影函数在眼睛定位上的不足之后,给出了最小邻域均值投影函数的描述. 第 3 节详细介绍利用本文提出的最小邻域均值投影函数实现眼睛的精确、鲁棒定位的方法. 具体实验结果的分析 与对比在第 4 节中给出.最后是结论.

\section{1 研究背景}

\section{1 积分投影函数}

积分投影函数(integral projection function,简称 IPF) 是最早出现的投影函数.我们用 $I(x, y)$ 表示数字图像 $I$ 在 $(x, y)$ 处的像素值,则图像沿投影线 $x=x_{0}$ 在区间 $\left[y_{1}, y_{2}\right]$ 上的垂直积分投影 $I_{v}\left(x_{0}\right)$ 和沿投影线 $y=y_{0}$ 在区间 $\left[x_{1}, x_{2}\right]$ 上的 水平积分投影 $I_{h}\left(y_{0}\right)$ 分别定义为

$$
I_{v}\left(x_{0}\right)=\sum_{y=y_{1}}^{y_{2}} I\left(x_{0}, y\right), I_{h}\left(y_{0}\right)=\sum_{x=x_{1}}^{x_{2}} I\left(x, y_{0}\right)
$$

与之相应的均值投影函数(mean projection function, 简称 MPF)由下式所定义:

$$
M_{v}\left(x_{0}\right)=\frac{1}{N_{v}} I_{v}\left(x_{0}\right), M_{h}\left(y_{0}\right)=\frac{1}{N_{h}} I_{h}\left(y_{0}\right)
$$

这里, $I_{v}\left(x_{0}\right)$ 和 $I_{h}\left(y_{0}\right)$ 由式(1)给出, $N_{v}, N_{h}$ 分别表示在投影线 $x=x_{0}$ 和 $y=y_{0}$ 上相应投影区间内的像素个数.MPF 与 IPF 具有相同的性质. 在图像内容发生较大跃变的区域,对应的 IPF 和 MPF 值将出现跃变,形成波谷或波峰.

\section{2 方差投影函数}

方差投影函数(variance projection function, 简称 VPF)最早由 Feng 和 Yuen ${ }^{[3]}$ 提出, 并被用于眼睛定位. 垂直和 水平方差投影函数 $V_{v}\left(x_{0}\right)$ 和 $V_{h}\left(y_{0}\right)$ 分别由下式所定义:

$$
V_{v}\left(x_{0}\right)=\frac{1}{N_{v}} \sum_{y=y_{1}}^{y_{2}}\left[I\left(x_{0}, y\right)-M_{v}\left(x_{0}\right)\right]^{2}, V_{h}\left(y_{0}\right)=\frac{1}{N_{h}} \sum_{x=x_{1}}^{x_{2}}\left[I\left(x, y_{0}\right)-M_{h}\left(y_{0}\right)\right]^{2}
$$

这里, $M_{v}\left(x_{0}\right)$ 和 $M_{h}\left(y_{0}\right)$ 的定义见式(2).VPF 考察的是图像灰度值变化的剧烈程度. 它对灰度变化剧烈的瞳孔 区域将产生明显的波峰.而在灰度变化较为均匀的皮肤区域,则响应较小.因此,它比较适用于眼睛的定位. 


\section{3 混合投影函数}

IPF 反映灰度均值的变化, 而 VPF 反映方差的变化, 二者具有互补性. 耿新等人 ${ }^{[5]}$ 将二者结合起来,提出了混 合投影函数(hybrid projection function, 简称 HPF). 垂直和水平混合投影函数 $H_{v}\left(x_{0}\right)$ 和 $H_{h}\left(y_{0}\right)$ 分别定义为

$$
H_{v}\left(x_{0}\right)=\frac{1}{2} M_{v}^{\prime}\left(x_{0}\right)+\frac{1}{2}\left(1-V_{v}^{\prime}\left(x_{0}\right)\right), H_{h}\left(y_{0}\right)=\frac{1}{2} M_{h}^{\prime}\left(y_{0}\right)+\frac{1}{2}\left(1-V_{h}^{\prime}\left(y_{0}\right)\right)
$$

其中, $M_{v}^{\prime}\left(x_{0}\right), M_{h}^{\prime}\left(y_{0}\right), V_{v}^{\prime}\left(x_{0}\right), V_{h}^{\prime}\left(y_{0}\right)$ 分别是将式(2)、式(3)归一化到 $[0,1]$ 区间的结果. 由于眼睛区域具有低均值、 高方差的特性,为了避免产生 IPF 和 VPF 作用相抵的情况, 需要对方差投影的结果取补.

\section{2 最小邻域均值投影函数}

投影的一个重要应用就是眼睛定位. 它通过水平和垂直投影分别检测瞳孔的纵坐标和横坐标,从而获得眼 睛的位置.但是,由于受额发、阴影等低灰度片状噪声的影响,通常不能保证投影的结果在瞳孔处形成全局最小 值. 为了应对这种情况, 通常的做法是通过检测投影结果中的波谷(局部极小值)形成眼睛的候选位置,再通过一 定的规则进行篮选. 理想的投影函数需要能够抑制片状噪声, 在眼睛处形成唯一和显著的波谷. 否则, 就容易出 现误检和漏检.

\section{1 传统投影函数的不足}

IPF 是对当前投影线上所有灰度值求和的结果.这种全局运算对片状噪声十分敏感.反映在眼睛定位上, 就 是当出现头发、阴影以及化妆等低灰度因素的干扰时, 投影波形会产生众多的波谷, 增大了误检的概率. 同样, 这 些因素也会影响 VPF 和 HPF 的结果. 如图 1 所示, 其中, 左上角的眼区图像受到了额发的干扰,因此,IPF,VPF 和 $\mathrm{HPF}$ 的投影结果都产生、形成了众多的波谷,这就使得确定瞳孔位置的操作变得十分困难.

另一方面,传统的投影方法是一种一维的搜索算法,其水平投影和垂直投影是两个互相独立的过程.对于人 脸图像来说,由于可能存在一定角度的平面内旋转,这样,水平投影得到的眼睛纵坐标就不够准确,如图 2 中的虚 线. 但现有的投影方法对这一问题却无能为力. 如果能将水平投影和垂直投影两者结合起来考虑, 在进行垂直投 影的同时对水平投影的结果进行必要的修正,则将极大地提高眼睛定位的精度.

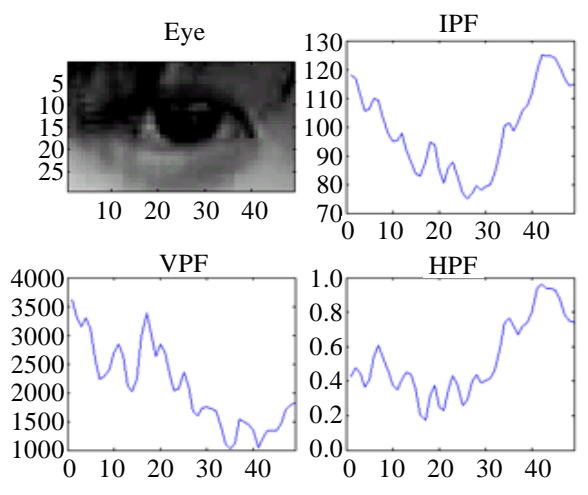

Fig.1 Disturbed eye image and vertical IPF, VPF and HPF results

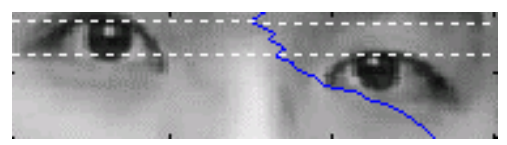

Fig.2 Height location error caused by face rotation

图 1 受干扰的眼区图像及垂直 IPF,VPF 和 HPF 的投影结果 图 2 人脸旋转造成眼睛高度定位误差

\section{2 最小邻域均值投影函数}

基于以上分析, 本文提出最小邻域均值投影函数 (minimal neighborhood mean projection function,简称 MNMPF). 以 $\bar{I}(x, y)$ 表示图像中以 $(x, y)$ 点为中心的邻域窗口均值, 窗口的形状和大小可根据实验确定. 公式(5)和 公式(6)分别定义了垂直和水平最小邻域均值投影 MNM 与相应的最小值点位置记录值 $P$ :

$$
M N M_{v}\left(x_{0}\right)=\min _{y \in\left[y_{1}, y_{2}\right]} \bar{I}\left(x_{0}, y\right), P_{v}\left(x_{0}\right)=\arg \min _{y \in\left[y_{1}, y_{2}\right]} \bar{I}\left(x_{0}, y\right)
$$




$$
\operatorname{MNN}_{h}\left(y_{0}\right)=\min _{x \in\left[x_{1}, x_{2}\right]} \bar{I}\left(x, y_{0}\right), P_{h}\left(y_{0}\right)=\arg \min _{x \in\left[x_{1}, x_{2}\right]} \bar{I}\left(x, y_{0}\right)
$$

MNMPF 不同于传统的投影函数,主要表现在它的局部选择性和具有二维搜索功能上. 由定义可 知,MNMPF 是以当前投影线上求各点邻域均值最小值的运算代替传统的积分、方差运算, 并跟踪记录了最小值 对应邻域窗口中心的 $x$ 或 $y$ 坐标值.MNMPF 特别适合于图像中前景对象数较少且灰度值较背景低很多的情况. 例如,用于眼区中睲孔的精确定位. 它的邻域求平均运算相当于先对图像作了平滑滤波,因此不易受随机噪声的 干扰. 同时, 由于以求最小值的局部选择性代替了求积分的全局累加性, 这使得它对阴影、头发这种大片低灰度 噪声干扰也具有良好的抑制能力 (如图 3 所示). 同时, 在利用垂直投影确定瞳孔的 $x$ 坐标时, 对每个最小邻域均值 点记录了其 $y$ 坐标, 从而可以形成最小值点的跟踪线(如图 4 所示). 如果这些最小值点中包含真实的瞳孔, 则可以 用所记录的 $y$ 坐标对之前水平投影的结果进行修正. 这使得水平投影和垂直投影不再是两个孤立的过程.
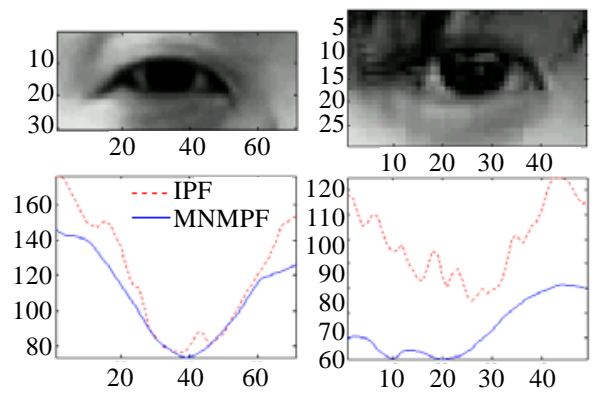

Fig.3 Vertical projection with IPF (dotted line) and MNMPF (solid line)

图 $3 \mathrm{IPF}$ (点线)与 $\mathrm{MNMPF}$ (实线)的垂直投影结果

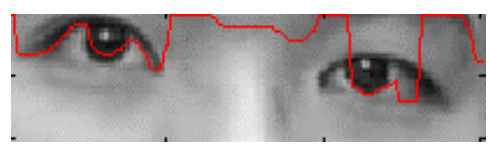

Fig.4 The tracing line for points with minimal neighborhood means (solid line)

图 4 最小邻域均值点的跟踪线(实线)

\section{3 眼睛定位}

为了缩小考察范围, 需要得到仅包含眼睛的图像, 即眼睛窗口. 为此, 我们对原图像先进行人脸检测 (face detection)与人脸分割(face segmentation). 我们采用 Viola 等人 ${ }^{[20]}$ 的基于 AdaBoost 的人脸检测方法.AdaBoost 是 一种统计的方法, 这样检测到的人脸所包含的面部区域较为一致(如图 5(a)所示).这种一致性让我们对面部各器 官所处位置能够有较好的估计 (图 5(b)中的虚线), 有助于人脸分割. 分割采用的是积分投影函数, 水平投影在眉 毛、眼睛、鼻孔、嘴巴的位置出现明显的波谷而被检测出来(图 5(b)中的实线), 再依据先前估计的位置就可以 确定各器官的真实位置.最后,利用器官大小比例及人脸对称性, 就可以得到眼睛窗口(图 5(c)中的方框).

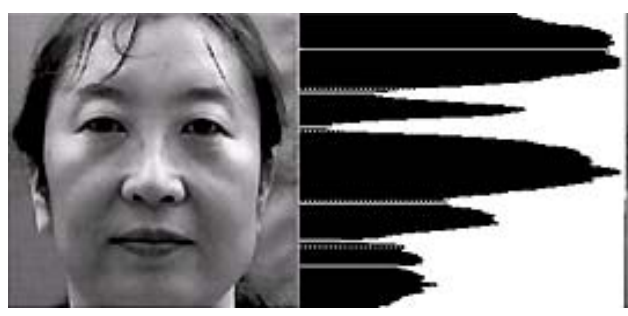

(a) Detected face image (a) 检测的人脸图像 (b) Horizontal projection (b) 水平投影

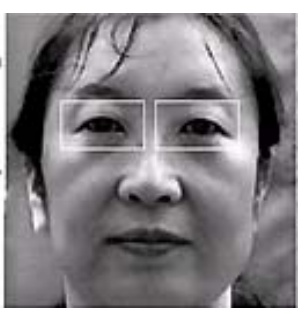

(c) Eye windows

(c) 眼睛窗口

Fig.5 Face detection and face segmentation 图 5 人脸检测与人脸分割

得到的眼睛窗口中可能包含眉毛, 会对眼睛定位带来干扰. 我们对窗口高度的 $1 / 4$ 以上的部分进行搜索, 将 其中的低灰度值置为平均灰度值, 以去除眉毛.下面就可以利用 MNMPF 来定位瞳孔. 具体步骤如下:

（1）根据眼睛窗口的大小,选取合适的邻域窗口大小计算 MNMPF,并记录最小值点的位置; 
(2) 在形成的投影波形上检测波谷,并取全局最小值的 1.5 倍作为阈值 $T$;

(3) 若只检测到一个波谷,则直接将其判定为瞳孔点;否则,转到步骤 4;

(4) 此时, 有一个以上的候选瞳孔点. 考察每一个灰度值小于 $T$ 的波谷点, 以其对应图像点为中心取一邻 域作“特征眼(eigeneyes)”[21]验证,取 PCA 重建误差最小的点作为瞳孔.

\section{4 实 验}

实验采用的数据来源于 CAS-PEAL ${ }^{[22]}$ 数据库的 Normal 子集和 BioID ${ }^{[23]}$ 数据库. 这两组数据有着截然不同 的特点:前者是在受控条件下拍摄的,包含了 1040 个人的正面、中性表情和控制光照条件下的图像,每个人 1 幅.被摄对象全部是亚洲人.图像细节较为清晰,但受到额发、阴影、化妆等因素的干扰.检测的人脸大小约为 $220 \times 220$; 后者是非受控条件下的人脸图像,包含了 23 个人的共 1521 张正面图像,主要是欧美人.图像不但在光 照条件、表情、人脸大小上均有较大变化, 而且拍摄对象还有诸如闭眼等非配合的行为. 检测的人脸大小约为 $127 \times 127$.这两组数据中的一些典型的人脸图像如图 6 所示.

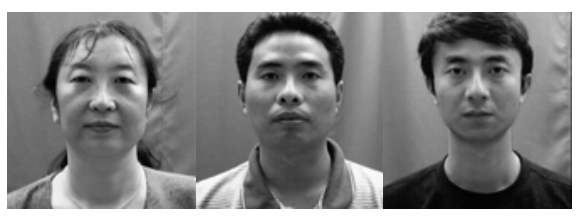

(a) Face samples from CAS-PEAL database (a) CAS-PEAL 数据库中的人脸

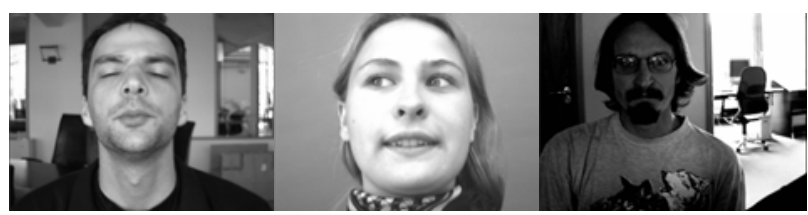

(b) Face samples from BioID database

(b) BioID 数据库中的人脸

Fig.6 Face samples from experimental datasets

图 6 实验数据集中的人脸

我们采用了 Jesorsky 等人的度量标准 ${ }^{[24]}$. 文献[5]也采用了此标准. 定义定位误差 err 为

$$
\text { err }=\frac{\max \left(d_{l}, d_{r}\right)}{d_{l r}}
$$

其中, $d_{l r}$ 是图像上左右瞳孔手工标定点之间的距离, $d_{l}$ 和 $d_{r}$ 分别是左右瞳孔手工标定点与算法定位点的距离. Jesorsky 等人提出,如果 $e r r<0.25$, 则可以认为定位正确,因为通常情况下,此时的误差距离不到眼睛宽度的一半, 低于这一误差被认为是可接受的 ${ }^{[24]}$. 我们采用了更为严格的标准,即 $e r r<0.1$ 视为定位正确,此时, 定位误差小于 瞳孔半径.err 值越小, 表示定位点与标定点间的距离越小,结果越精确.表 1 比较了 IPF,HPF 和 MNMPF 在两种 不同标准下的定位正确率以及平均的定位误差(在 CAS-PEAL 数据库上的实验结果). 从表中可见,当正确标准 由 0.25 提高到 0.1 时, MNMPF 的正确率几乎没有受到影响, 而且其平均误差也较其他两种投影函数小得多. 这 说明,MNMPF 在定位精度上优于传统的投影函数.

Table 1 Experiment results on CAS-PEAL database with the three projection functions

表 13 种投影函数在 CAS-PEAL 数据库上的实验结果

\begin{tabular}{cccc}
\hline & Correct rate (with err $<0.25$ ) & Correct rate (with err $<0.1$ ) & Average err of all the results \\
\hline IPF & 97.02 & 76.35 & 0.0957 \\
HPF & 98.52 & 88.75 & 0.0744 \\
MNMPF & 99.62 & 99.33 & 0.0471 \\
\hline
\end{tabular}

为了更直观地反映 MNMPF 定位的精确性,我们计算了算法定位点与手工标定点之间的距离. 图 7 的横坐 标表示图像上两点之间的距离, 以像素为单位; 对应的纵坐标表示定位误差低于这一像素的结果在所有结果中 所占的百分比(频率), 所得到的曲线越陡表示定位误差越小.3 条线从上到下依次为 MNMPF,HPF 和 IPF, 可以看 出, MNMPF 有 $95 \%$ 以上的检测结果都低于 6 个像素(实验图像瞳孔平均半径约为 8 个像素), 其性能较其他两个 投影函数提升很多,而这种性能的提高并没有带来计算时间的增加. 在每条投影线上计算 MNMPF 只要 1 次循 环, 而 HPF(或 VPF)需要 2 次. 实际上, 在多种眼睛定位方法中, 投影只是作为预处理以得到眼睛位置的初始估计, 再利用一些局部搜索的方法得到更精确的瞳孔中心位置(但这将使得计算时间大为增加). 我们在 MNMPF 投影 
的结果基础上采用文献[10]的方法进一步细化,定位精度提高到 3 个像素以内.

为了验证 MNMPF 对光照变化的鲁棒性, 我们选取了 CAS-PEAL 数据库的 Lighting 子集下 10 个人的各种 光照条件下的人脸图像共 151 幅进行眼睛定位. 在 $e r r<0.1$ 的标准下,正确定位了 149 幅. 图 8 显示了该数据库中 编号为 46 的被试者在 3 种极端光照条件下的眼睛定位结果.

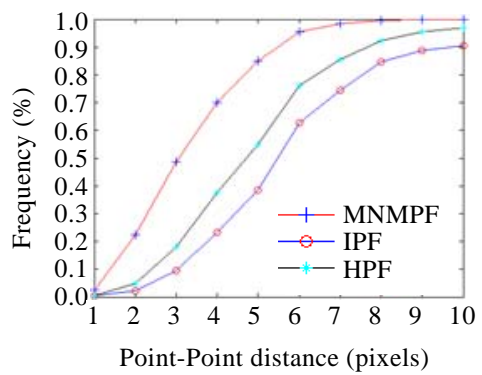

Fig.7 The frequency of point-to-point distance lower than certain pixel

图 7 定位误差低于某像素的频率图

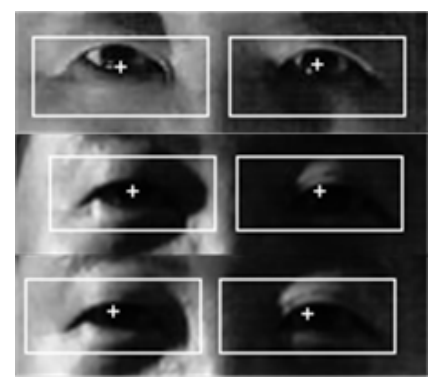

Fig.8 MNMPF results under bad lighting conditions

图 8 不良光照条件下 MNMPF 的定位结果

为了考察 MNMPF 在非受控条件下拍摄的人脸图像上的性能, 我们也在 BioID 数据库上作了实验, 并与文 献[5]的基于 HPF 的方法作比较,结果见表 2. 我们的结果要优于文献[5]的结果.并且,我们的结果与文献[18]的基 于 SVM 和方差滤波的结果(96.8\%)相比,性能相仿,而计算复杂度都大为降低.

Table 2 Experiment result on BioID with MNMPF

表 2 MNMPF 在 BioID 数据库上的实验结果

\begin{tabular}{ccc}
\hline & Correct location rate (with err $<0.25)$ & Average location err of correct results \\
\hline MNMPF & 96.35 & 0.0722 \\
HPF (Ref.[5]) & 94.81 & 0.0870 \\
\hline
\end{tabular}

\section{5 结 论}

本文提出了一种投影函数:最小邻域均值投影函数(MNMPF), 并且针对 MNMPF 的特点, 设计了一套以投影 为核心的眼睛定位的方法. 通过在两个较大的公开的数据库上进行的实验表明,基于 MNMPF 的定位方法达到 了较高的正确率和精确度. 该方法即可以作为一种简单、精确的眼睛定位方法,并且因其计算复杂度很低,也可 以与一些已有方法结合, 进行更为精细的定位. 本文的贡献在于突破了传统投影方法的线性和全局的思维方式, 首次将非线性和局部选择性引入了投影函数. 实际上, 针对不同的应用场合, 可以将其他的非线性函数引入到投 影函数中来.而这也正是我们下一步工作的内容.

致谢 本文使用了 CAS-PEAL 数据库,在此表示感谢.

\section{References:}

[1] Brunelli R, Poggio T. Face recognition: Features versus templates. IEEE Trans. on Pattern Analysis and Machine Intelligence, 1993, 15(10):1042-1052.

[2] Wu H, Yokoyama T, Pramadihanto D, Yachida M. Face and facial feature extraction from color image. In: Proc. of the 2nd IEEE Int'l Conf. on Automatic Face and Gesture Recognition. 1996. 345-350. http://ieeexplore.ieee.org/iel3/4096/12122/00557289.pdf

[3] Feng GC, Yuen PC. Variance projection function and its application to eye detection for human face recognition. Pattern Recognition Letters, 1998,19(9):899-906.

[4] Kumar RT, Raja SK, Ramakrishnan AG. Eye detection using color cues and projection functions. In: Proc. of the IEEE Int'l Conf. on Image Processing. 2002. 337-340. http://ieeexplore.ieee.org/xpls/abs_all.jsp?arnumber=1038974

[5] Geng X, Zhou ZH, Chen SF. Eye location based on hybrid projection function. Journal of Software, 2003,14(8):1394-1400 (in Chinese with English abstract). http://www.jos.org.cn/1000-9825/14/1394.htm 
[6] Kothari R, Mitchell JL. Detection of eye locations in unconstrained visual images. In: Proc. of the IEEE Int'l Conf. on Image Processing. 1996. 519-522. http://ieeexplore.ieee.org/xpls/abs_all.jsp?arnumber=560546

[7] Kawaguchi T, Hidaka D, Rizon M. Detection of eyes from human faces by Hough transform and separability filter. In: Proc. of the IEEE Int'l Conf. on Image Processing. 2000. 49-52. http://ieeexplore.ieee.org/Xplore/login.jsp?url=/iel5/7221/19490/00900889. pdf?arnumber $=900889$

[8] D’Orazio T, Leo M, Cicirelli G, Distante A. An algorithm for real time eye detection in face images. In: Proc. of the IEEE Int'l Conf. on Pattern Recognition. 2004. 278-281. http://ieeexplore.ieee.org/xpl/freeabs_all.jsp?arnumber=1334521

[9] Cao B, Shan SG, Gao W, Zhao DB. Localizing the iris center by region growing search. In: Proc. of the IEEE Int'l Conf. on Multimedia and Expo. 2002. 129-132. http://ieeexplore.ieee.org/xpl/freeabs_all.jsp?arnumber=1035529

[10] Xu ZF, Shi PF. A robust and accurate method for pupil features extraction. In: Proc. of the IEEE Int'l Conf. on Pattern Recognition. 2006. 437-440. http://ieeexplore.ieee.org/Xplore/login.jsp?url=/iel5/11159/35820/01699907.pdf?temp=x

[11] Du G. Eye location method based on symmetry analysis and high-order fractal feature. In: Vision, Image and Signal Proc. 2006. 11-16. http://ieeexplore.iee.org/Xplore/login.jsp?url=/iel5/2200/33319/01576411.pdf?arnumber=1576411

[12] Huang J, Wechsler H. Eye detection using optimal wavelet packets and radial basis functions (rbfs). Int'l Journal of Pattern Recognition and Artificial Intelligence, 1999,13(7):1009-1026.

[13] Feris RS, Gemmell J, Toyama K, Kruger V. Hierarchical wavelet networks for facial feature localization. In: Proc. of the IEEE Int'l Conf. on Automatic Face and Gesture Recognition. 2002. 118-123. http://citeseer.ist.psu.edu/663101.html

[14] Du G, Su F, Cai AN. Eye location under various illumination conditions. In: Proc. of the Int'l Multi-Conf. on Computing in the Global Information Technology. 2006. 12-16. http://ieeexplore.ieee.org/Xplore/login.jsp?url=/iel5/4124012/4124013/04124031.pdf

[15] Peng W, Green MB, Qiang J, Wayman J. Automatic eye detection and its validation. In: Proc. of the IEEE Conf. on Computer Vision and Pattern Recognition. 2005. 164-171. http://ieeexplore.ieee.org/xpl/freeabs_all.jsp?arnumber=1565482

[16] Ma Y, Ding X, Wang Z, Wang N. Robust precise eye location under probabilistic framework. In: Proc. of the IEEE Int'l Conf. on Automatic Face and Gesture Recognition. 2004. 339-344. http://doi.ieeecomputersociety.org/10.1109/AFGR.2004.1301554

[17] Ian F, Bret F, Javier M. A generative framework for real time object detection and classification. Computer Vision and Image Understanding, 2005,98(1):182-210.

[18] Wang Q, Yang JY. Eye detection in facial images with unconstrained background. Journal of Pattern Recognition Research, 2006,1(1):55-62.

[19] Paola C, Raffaella L, Giuseppe L. Eye localization: A survey. 2006. http://homes.dsi.unimi.it/ lipori/publications. html

[20] Viola P, Jones M. Rapid object detection using a boosted cascade of simple features. In: Proc. of the IEEE Conf. on Computer Vision and Pattern Recognition. 2001. 511-518. http://ieeexplore.ieee.org/xpl/freeabs_all.jsp?arnumber=990517

[21] Pentland A, Moghaddam B, Starner T. View-Based and modular eigenspaces for face recognition. In: Proc. of the IEEE Conf. on Computer Vision and Pattern Recognition. 1994. 84-91. http://ieeexplore.ieee.org/Xplore/login.jsp?url=/iel2/977/7716/00323814. pdf?temp $=\mathrm{x}$

[22] Gao W, Cao B, Shan SG, Zhou DL, Zhang XH, Zhao DB. The CAS-PEAL large-scale Chinese face database and evaluation protocols. Technical Report, No. JDL_TR_04_FR_001, Joint Research \& Development Laboratory, CAS, 2004.

[23] The BioID face database. 2003. http://www.humanscan.de/support/downloads/facedb.php

[24] Jesorsky O, Kirchberg KJ, Frishholz RW. Robust face detection using the Hausdorff distance. In: Proc. of the Audio and Video based Person Authentication. 2001. 90-95. http://www.springerlink.com/content/ua5cq1ryt355al1b/

\section{附中文参考文献:}

[5] 耿新,周志华,陈世福.基于混合投影函数的眼睛定位.软件学报,2003,14(8):1394-1400. http://www.jos.org.cn/1000-9825/14/ 1394.htm

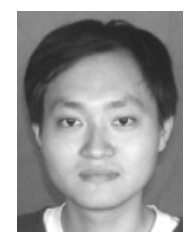

郑颖 $(1981-)$, 男,福建莆田人,博士生,主 要研究领域为图像处理,模式识别, 人脸 识别.

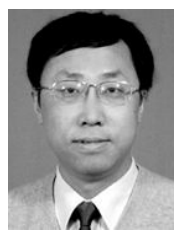

汪增福(1960-), 男, 博士, 教授, 博士生导 师,主要研究领域为视听觉信息处理,模式 识别,智能机器人. 